\begin{tabular}{|c||c|c|c|} 
Journal of Bangladesh Academy of Sciences \\
Journal homepage: http://www.bas.org.bd/publications/jbas.html
\end{tabular}

Research Article

\title{
Optimizing the wash time of the washing machine using several types of fuzzy numbers
}

Md. Azharul Islam and Md. Sahadat Hossain

Department of Mathematics, University of Rajshahi, Bangladesh

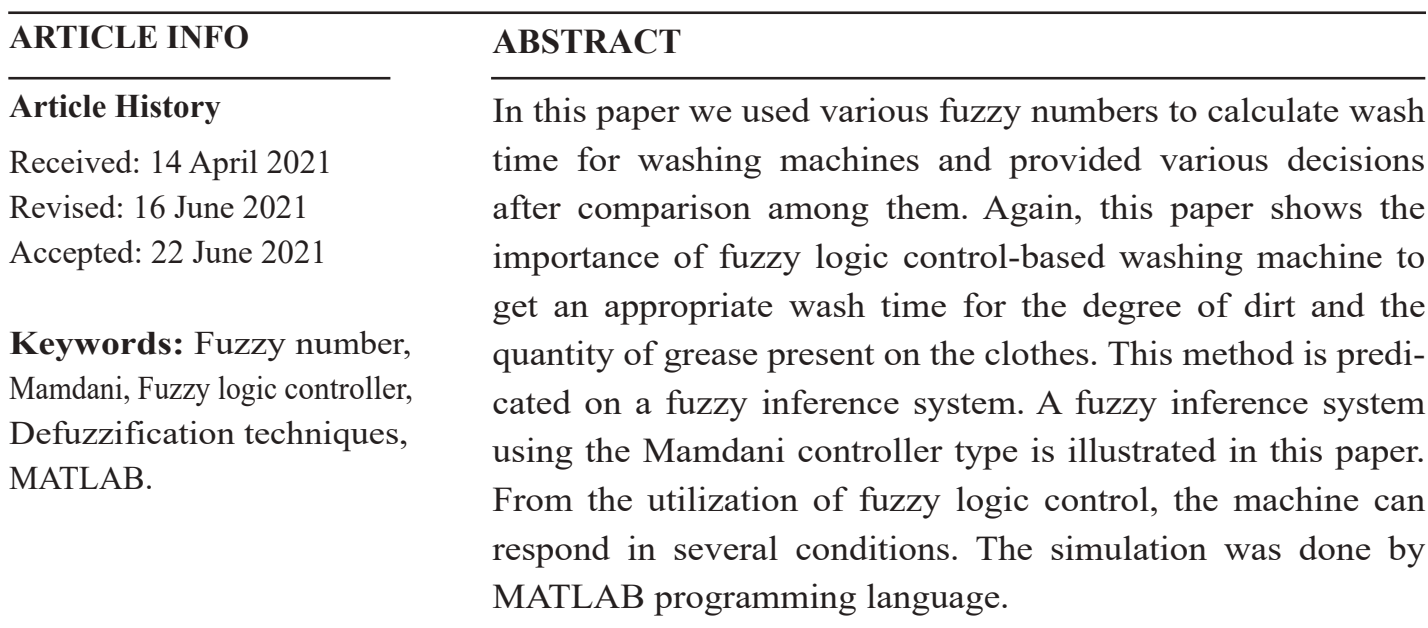

\section{Introduction}

The fuzzy set and fuzzy logic were first introduced by Zadeh (1965) in 1965. After that, for the first time, Mamdani (1974) demonstrated its application in 1974. Then, Alhanjouri et al. (2013) contributed a lot about it, moreover, several researchers, namely, Aggarwal (2011); Akram et al. (2014) and calculated wash time for washing Machines using fuzzy logic. Further, many researchers, namely Miah et al. (2018); Amin et al. (2020), and Mahbub et al. (2019) are also working on fuzzy set.

This paper demonstrates the process of controlling the wash time using FLC. On top of that, it elucidates the processes which can be applied to get the expected wash time for the dirtiness of clothes and the amount of grease on the clothes. The process formulated here is completed considering the principle of sorting out non-precise input from the sensors, which subject them to fuzzy arithmetic. Thus, obtaining a crisp value of the wash time. This paper will explain that this process can be used practically for further automation of the washing machine.

\section{Basic Definition}

This section presents the notation and some basic definitions, say the triangular fuzzy number, trapezoidal fuzzy number, and linguistic variable used throughout the paper.

\section{Triangular Fuzzy Number (TFN)}

Suppose A is a fuzzy number. Then the membership function $\mu_{A}(x)$ of the triangular fuzzy number is characterized by three parameters, and is defined as (Dahmardeh et al., 2011). 


$$
\mu_{A}(x)=\left\{\begin{array}{cc}
\frac{x-a_{1}}{a_{2}-a_{1}}, \text { if } a_{1} \leq x \leq a_{2} \\
\frac{a_{2}-x}{a_{3}-a_{2}}, \text { if } a_{2} \leq x \leq a_{3} \\
0, & \text { otherwise }
\end{array}\right.
$$

The graphical representation of TFN is present in Fig 1.

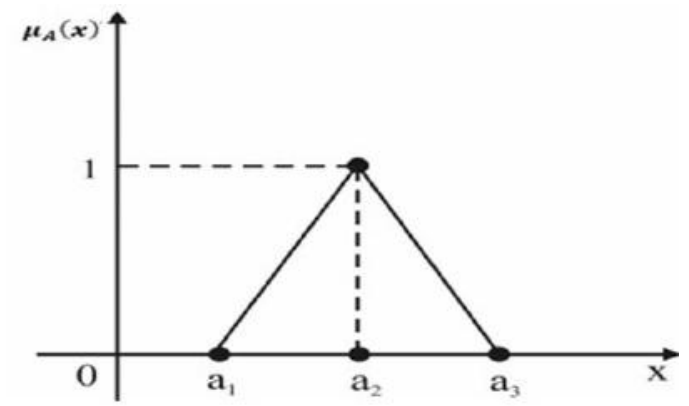

\section{Trapezoidal Fuzzy Number (TrFN)}

Suppose A is a fuzzy number. Then the membership function $\mu_{A}(x)$ of trapezoidal fuzzy number is characterized by four parameters $a_{2}, a_{1}, a_{3}$, and $a_{4}$ is defined as (Dahmardeh et al., 2011).

$$
\mu_{A}(x)=\left\{\begin{array}{cc}
\frac{x-a_{1}}{a_{2}-a_{1}}, & \text { if } a_{1} \leq x \leq a_{2} \\
1, & \text { if } a_{2} \leq x \leq a_{3} \\
\frac{a_{4}-x}{a_{4}-a_{3}}, & \text { if } a_{3} \leq x \leq a_{4} \\
0, & \text { otherwise }
\end{array}\right.
$$

The graphical representation of the trapezoidal fuzzy number is present in Fig. 2.

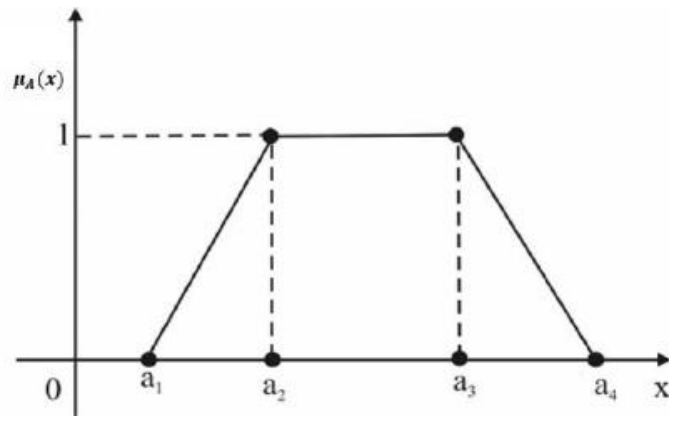

Fig. 2. Trapezoidal Fuzzy Number.

\section{Linguistic Variable}

A linguistic variable represents this type of variable whose values are words or sentences in an artificial or natural language (Isables et al., 2012).

\section{Defuzzification Techniques}

The process that derives control output from the combination of input and output membership functions, including fuzzy rule base, which is either vague or fuzzy, is called fuzzy inference. The fuzzification method is supposed to convert the fuzzy output, back to the crisp or classical output the control objective. The operation defuzzification can be performed in different ways. Trapezoidal fuzzy numbers, mixed fuzzy numbers, supporting interval's length, clipped triangular's height, and mixed closeness to central triangular fuzzy numbers. etc., are taken under consideration during this method is carried out. Some of the methods are named by

1. Mean of Maxima (MOM) Method;

2. Center of Sum (COS) Method;

3. Center of Gravity (COG) Method.

\section{Mean of Maxima (MOM) Method}

The easiest way of defuzzification is to take the nearby crisp value with the highest membership function. The arithmetic average of all mean values of the intervals that contain fuzzy set, including zero-length intervals, is called the mean of maxima method. The general equation of this method is given by (Mukaidono, 2001).

$$
x=\frac{\sum_{x_{i \in M}} x_{i}}{|M|}
$$

Where $\quad M=$ height of the fuzzy set and $M \mid=$ cardinality of the fuzzy set $M$. 


\section{Center of Sum (COS) Method}

Of all the techniques prevalent in the process of defuzzification, this one takes the lead. This method adopts a device of counting the overlapping area twice. The defuzzified value $x$ is defined as (Bai et al., 2006; Mogharreban et al., 2006).

$$
x=\frac{\sum_{i=1}^{n} A_{i} \cdot \bar{x}_{l}}{\sum_{i=1}^{n} A_{i}}
$$

Here, $A_{i}$ represents the firing area of $i^{\text {th }}$ rules and $n$ is the total number of rules fired, and represents the center of area $\bar{x}_{l}$.

\section{Center of Gravity (COG) Method}

The "centroid method", also known as the "center of gravity" or "center of area" method, is a technique that is most commonly employed and familiar for defuzzification. It reduces the area to smaller regions, and a combined operation is performed to obtain the final output. It is stated by the expression below:

(Bai et al., 2006; Mogharreban et al., 2006)

$$
x=\frac{\sum_{i=1}^{n} x_{i} \cdot \mu\left(x_{i}\right)}{\sum_{i=1}^{n} \mu\left(x_{i}\right)}
$$

Here, $\mathrm{n}$ represents the number of elements in the sample, i.e., $x_{i}$ is the elements, and $\mu\left(x_{i}\right)$ are their corresponding membership functions.

\section{Proposed Design of a FLC of Washing Machine General Idea of Fuzzy Logic Control System for Washing Machine}

In the past, before starting the washing process, the type of wash, the amount of water, and the duration of the cycle had to be selected manually. Now a days, FLC is employed to make this process automated. It is to be noted that for saving water, the FLC is a very efficient process. Machines that employ fuzzy control can calculate the effect of several variables and decide the right settings for the wash. The weight of clothes, the type of dirt, water, and electricity required for optimal wash are variables that fuzzy logic evaluates. Fuzzy Logic Structure: The basic elements of fuzzy logic; Fuzzy inputs, outputs, rules, and defuzzification, are shown in Fig. 3.

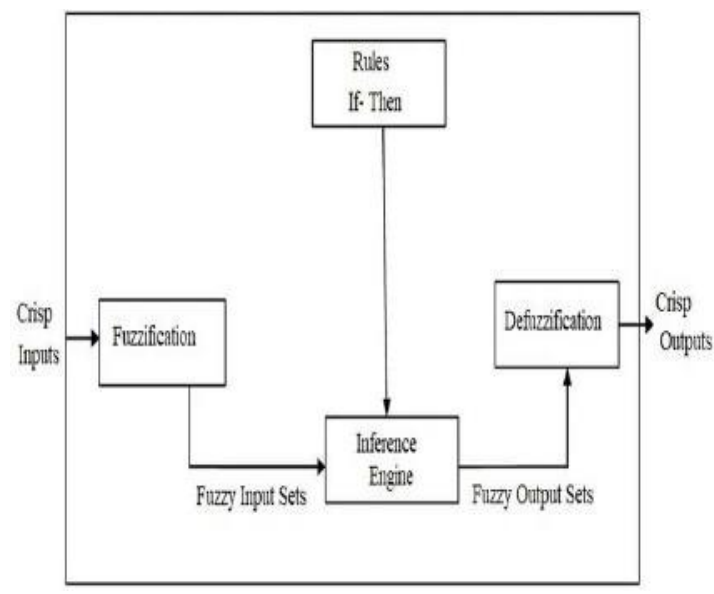

Fig. 3. Fuzzy Logic System

Fuzzy collects information from a system containing a normal language and transforms it into values. The values of input quantities related to membership functions which are written in the form of words such as small, smallest (Saade et al., 2004).

Input and output variables of fuzzy systems are used, and these values, i.e., less, much, hot, and cold, are chosen. Afterward, a relationship between input and output is developed based on the rules developed.

Outputs are created by applying fuzzified inputs and the determined rules to use them. In a real system, those fuzzified outputs must be converted into absolute values. This whole process is known as defuzzification (Saade et al., 2004).

\section{System Design}

The working principle of the Fuzzy inference system or FIS can be divided into four steps: 
Step-1: Firstly, fuzzification of the input variables is carried out. Here, crisp inputs are transformed into fuzzy inputs. Hence, membership function is obtained, representing those crisp inputs into fuzzy numbers depending on their linguistic terms.

Step-2: Secondly, the rule evaluation is carried out to evaluate this process.

Step-3: Next, the aggregation of the outputs is carried out.

Step-4: Finally, defuzzification is carried out where the fuzzy inputs change into crisp outputs.

In this paper, we use the Mamdani, Mean of Maxima, and Centroid method for washing machine's design development. This FLC for consists of two linguistic inputs i.e.

1. Present degree of dirt on the clothes and

2. Present amount of grease on the clothes.

To obtain the wash time as an output, these two linguistic variables are used. Here, for acquiring wash time, 9 rules are executed in the FLC inference engine. For all linguistic inputs, and linguistic outputs triangular, trapezoidal, and mixed MF are used.

Fuzzy Logic Modeling of Washing Machine

To check the system continuously and execute the operation as well, fuzzy logic control is used. Here, the Mamdani method is used to calculate the time period with high accuracy.

\section{The MF of fuzzy inputs and output}

For each variable, we obtained the membership functions of the proposed fuzzy system, which includes two inputs and one output, as mentioned above. The washing machine system is shown in Fig. 4.

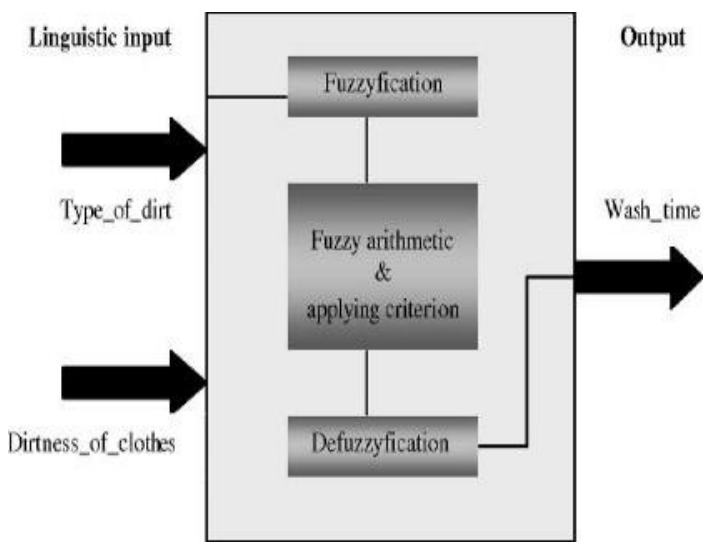

Fig. 4. FLC for Washing Machine

In this paper, several linguistic variables and their ranges regarding fuzzy inputs and output are illustrated as below:

\section{Fuzzy Inputs}

Usually, in fuzzy mathematics, crisp inputs can be converted into fuzzy inputs where the membership function represents crisp inputs based on their linguistic terms and ranges. Here, three linguistic terms such as small (SD), medium (MD), and large (LD) of present degree of dirt on the cloth are taken. Secondly, the present amount of grease on the clothes with 3 linguistic variables such as the amount of no grease (NG), medium amount of grease (MG), and a large amount of grease (LG) are taken. Now, the universe of discourse for the present degree of dirt and the present amount of grease is $[0,100]$. The MFs. corresponding to the linguistic values are represented by the following TFN, TrFN and mixed FN (i.e., Combine TFN, and TrFN). 


\section{Fuzzy Output}

The main purpose of this washing machine is to observe the wash time of these inputs and also divide them into 5 linguistic variables such as very short time period (VS), Short-time period (S), medium time period $(\mathrm{M})$, large time period (L) and very large time period $(\mathrm{VL})$ that belong to the universe $[0,60]$.

Now, the membership functions are in the following tables:

Table 1. Membership value of Input Variables

\begin{tabular}{ccc}
\hline $\begin{array}{c}\text { Linguistic } \\
\text { Value }\end{array}$ & Notation & $\begin{array}{c}\text { Numerical Range } \\
\text { (Normalized) }\end{array}$ \\
\hline
\end{tabular}

Linguistic Input Variable: Dirt

$\begin{array}{ccc}\text { Small Dirt } & \text { SD } & {[0,50]} \\ \text { Medium Dirt } & \text { MD } & {[0,100]} \\ \text { Large Dirt } & \text { LD } & {[50,100]}\end{array}$

Linguistic Input Variable: Grease

$\begin{array}{ccc}\text { No Grease } & \text { NG } & {[0,50]} \\ \text { Medium Grease } & \text { MG } & {[0,100]} \\ \text { Large Grease } & \text { LG } & {[50,100]}\end{array}$

Table 2. Membership value of output Variable.

Linguistic Output Variable: Wash Time

\begin{tabular}{ccc}
\hline $\begin{array}{c}\text { Linguistic } \\
\text { Value }\end{array}$ & Notation & $\begin{array}{c}\text { Numerical Range } \\
\text { (Normalized) }\end{array}$ \\
\hline Very Short & VS & {$[0,10]$} \\
Short & S & {$[0,25]$} \\
Medium & M & {$[10,40]$} \\
Large & L & {$[25,60]$} \\
Very Large & VL & {$[40,60]$} \\
\hline
\end{tabular}

Here in Fig. 5, 6, 7, 8, 9, 10, 11, 12, and 13 inputs and output membership functions of fuzzy logic shown below:

For Triangular Fuzzy Number:

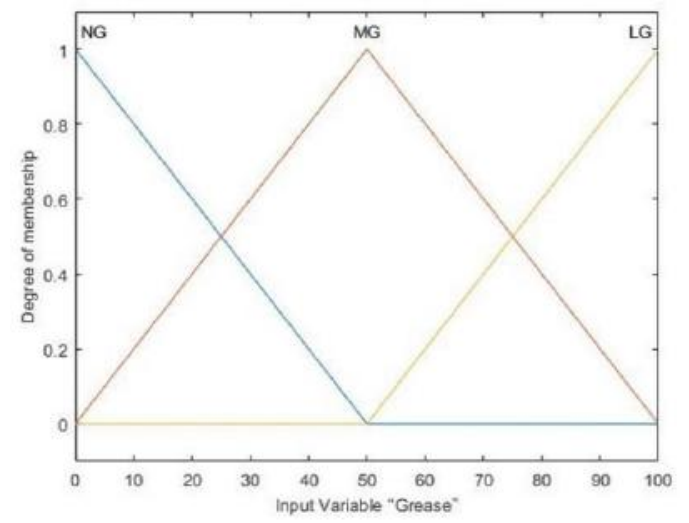

Fig. 5. Input variable dirt on the clothes

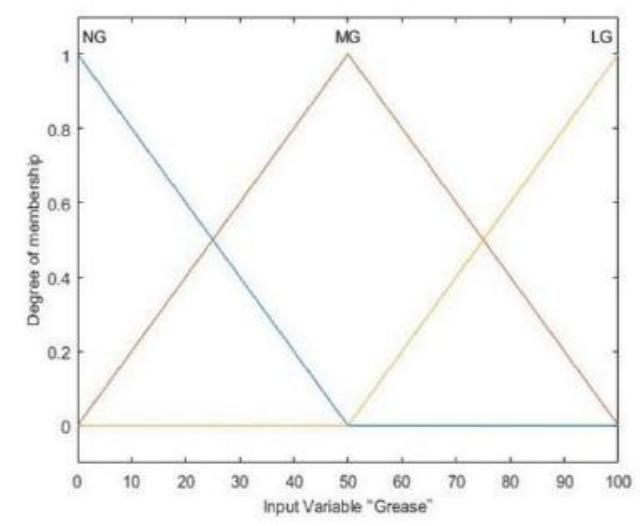

Fig. 6. Input variable grease on the clothes

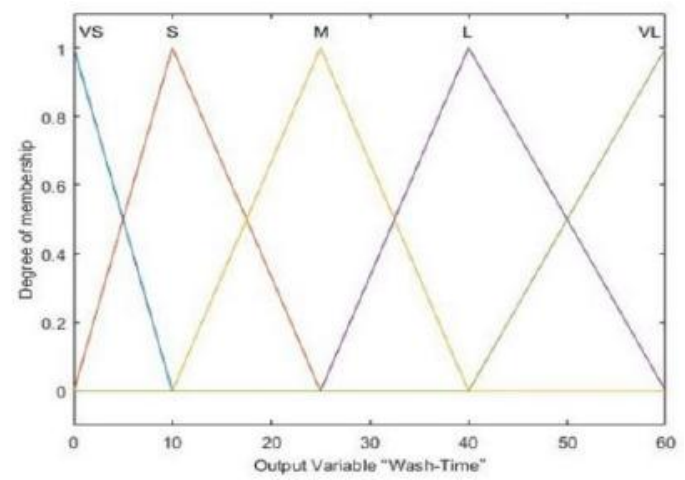

Fig. 7. Output variable wash time of TFN

For Trapezoidal Fuzzy Number: 
Islam et al./J. Bangladesh Acad. Sci. 45(1); 105-116: June 2021
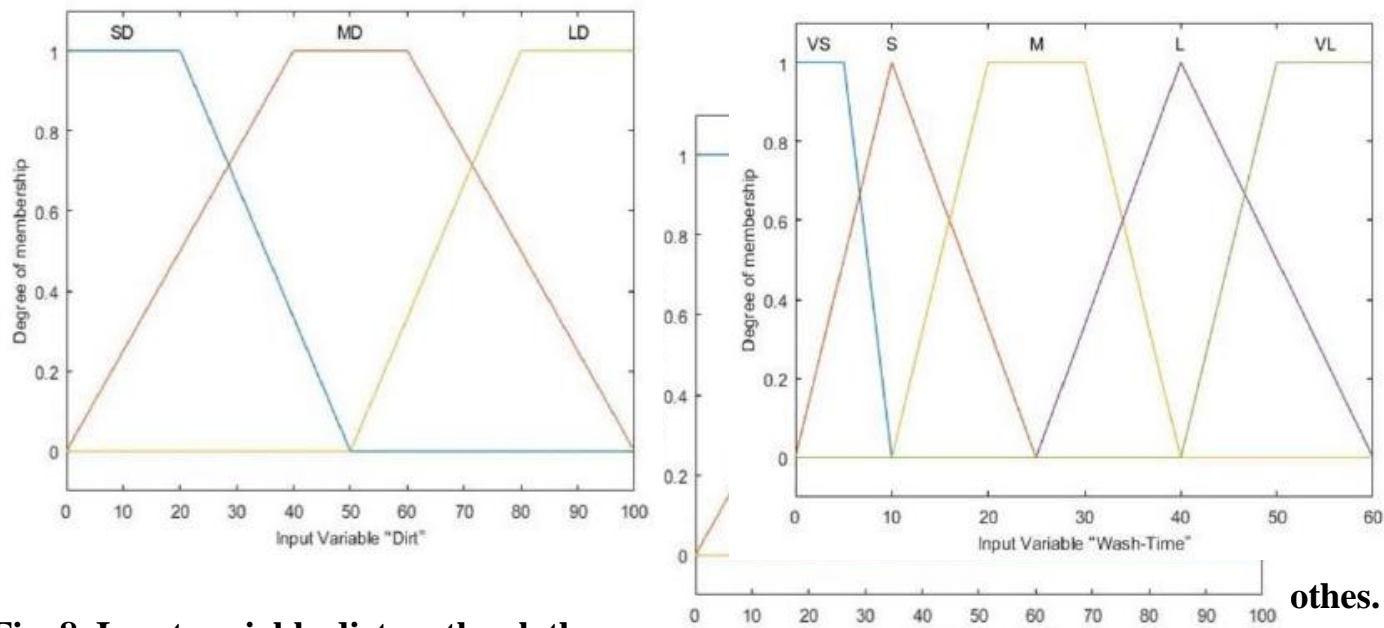

Fig. 8. Input variable dirt on the clothes.
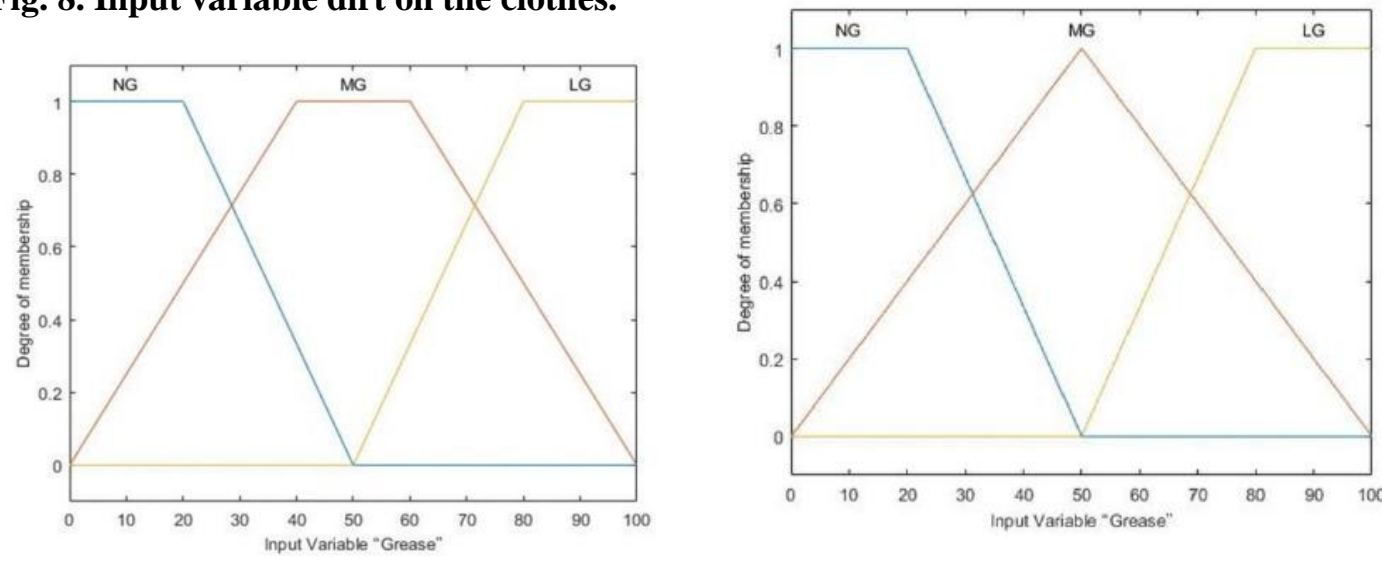

Fig. 9. Input variable grease on the clothes.

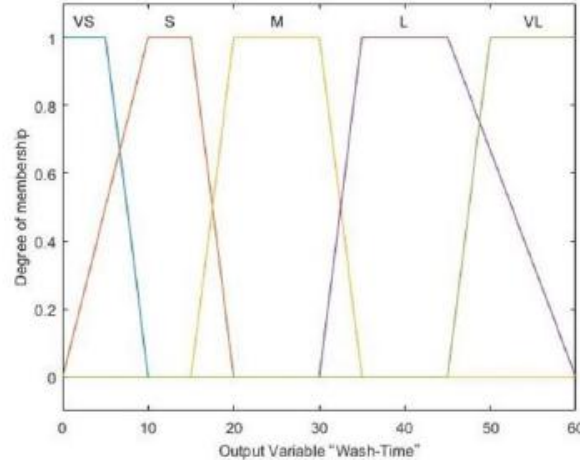

Fig. 10. Output variable wash time.

For Mixed (Combine TFN \& TrFN) Fuzzy Number:
Fig. 12. Input variable grease on the clothes.

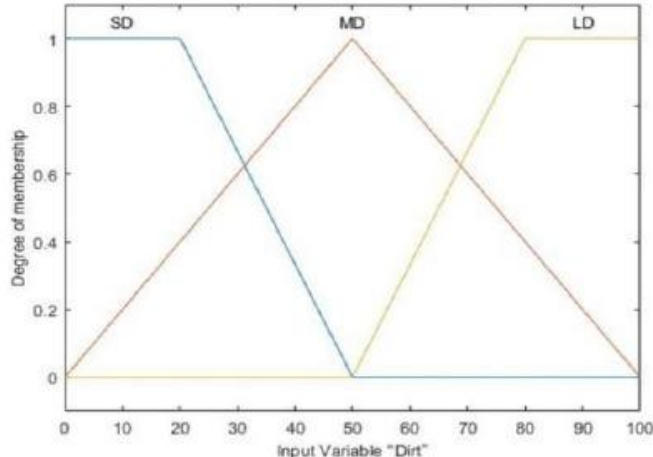

Fig. 13. Output variable wash time.

The working principle of the Mamdani-style fuzzy inference system is executed in four steps. 
In this Mamdani-style fuzzy inference, aggregation is Max, where the implication is Min. Here, are triangular, trapezoidal, and mixed fuzzy numbers are used.

\section{Fuzzification}

Firstly, a set of MF of linguistic variables is taken as inputs where input variables are words or sentences. To take any fuzzy or crisp output by fuzzification or defuzzification respectably, fuzzy MF are used.

Fuzzification is a process that provides fuzzy output. Here, crisp values are fuzzified for fuzzy output. The decision made by the logic controller is derived from the rules known as fuzzy rules. The fuzzy rules are applied in the FLC by selecting the appropriate sequence in the "If" and "then" rules based on natural language. It is designed to make any automated decision. It is formed by keeping the relationship between Input and output in mind. The input variables (Dirt and Grease) make a total of $3 * 3=9$ rules. The set of rules used in this paper are shown in table 3 .
Table 3. If-Then Rule base required is 9 rules.

\begin{tabular}{cccc}
\hline $\begin{array}{c}\text { Rule } \\
\text { Number }\end{array}$ & $\begin{array}{c}\text { The } \\
\text { degree of } \\
\text { dirt }\end{array}$ & $\begin{array}{c}\text { The } \\
\text { amount of } \\
\text { grease }\end{array}$ & $\begin{array}{c}\text { Wash } \\
\text { time }\end{array}$ \\
\hline 1 & SD & NG & VS \\
2 & SD & MG & S \\
3 & SD & LG & M \\
4 & MD & NG & S \\
5 & MD & MG & M \\
6 & MD & LG & L \\
7 & LD & NG & M \\
8 & LD & MG & L \\
9 & LD & LG & VL \\
\hline
\end{tabular}

\section{Rule Evaluation}

We assume the amount of dirt and the amount of grease is equal to 60 and 70, respectively. Then we get the following aggregation of the rule outputs.

\section{Aggregation of the rule output}

The aggregate control output procedure is shown in Figures 14, 15, and 16 for TFN, TrFN, and mixed FN.
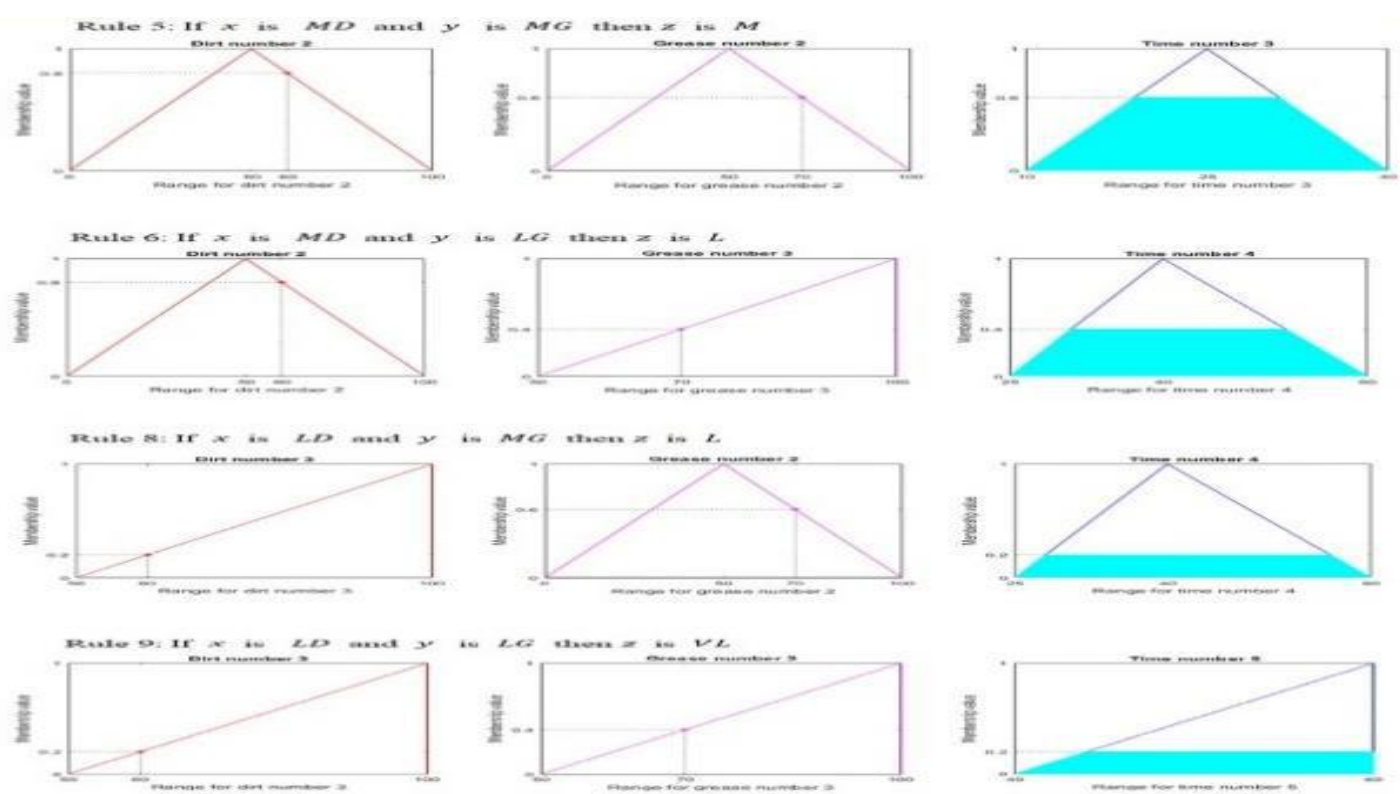

Fig.14. The aggregated the control output for TFN. 
Islam et al./J. Bangladesh Acad. Sci. 45(1); 105-116: June 2021
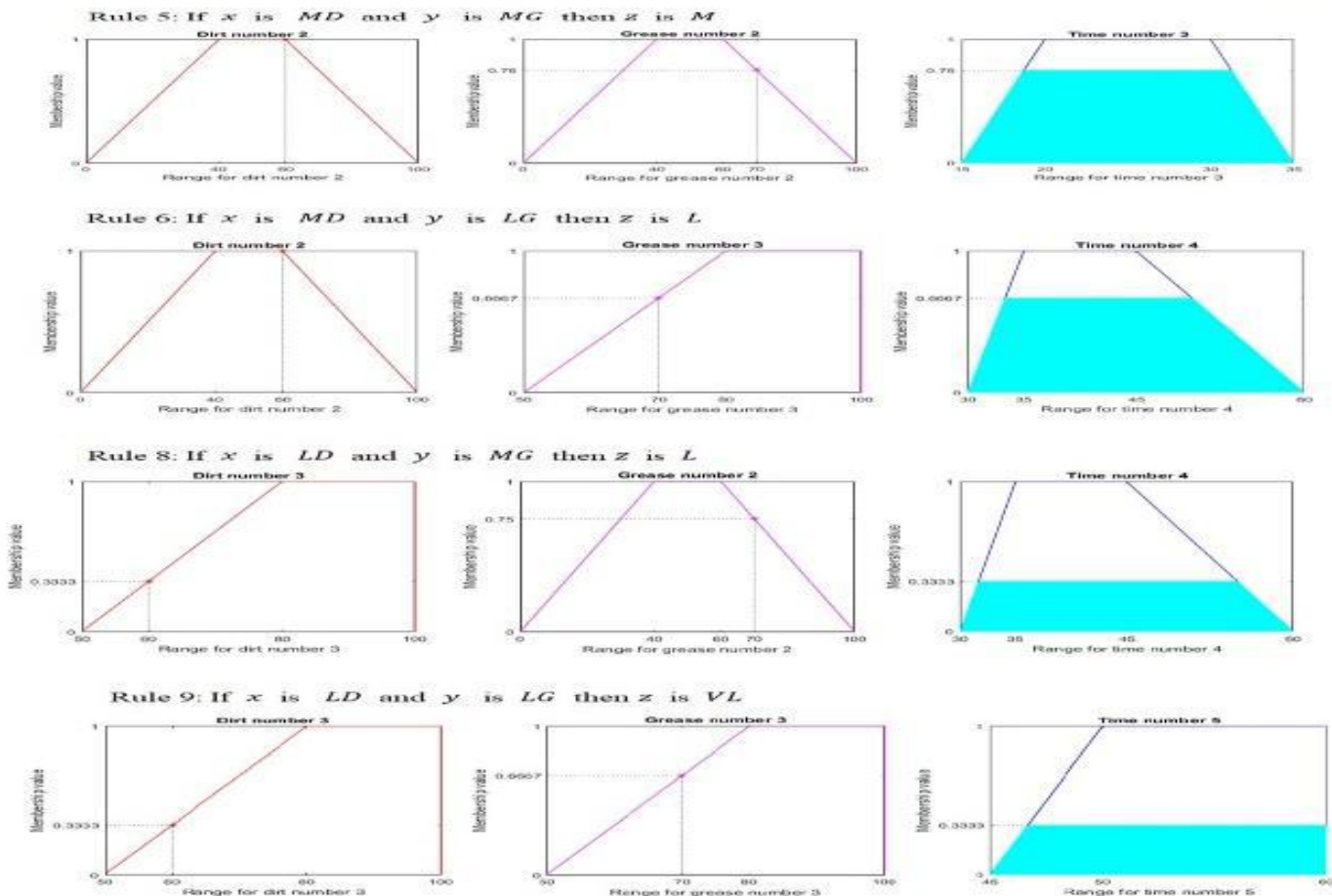

Fig. 15. The aggregated the control output for TrFN.
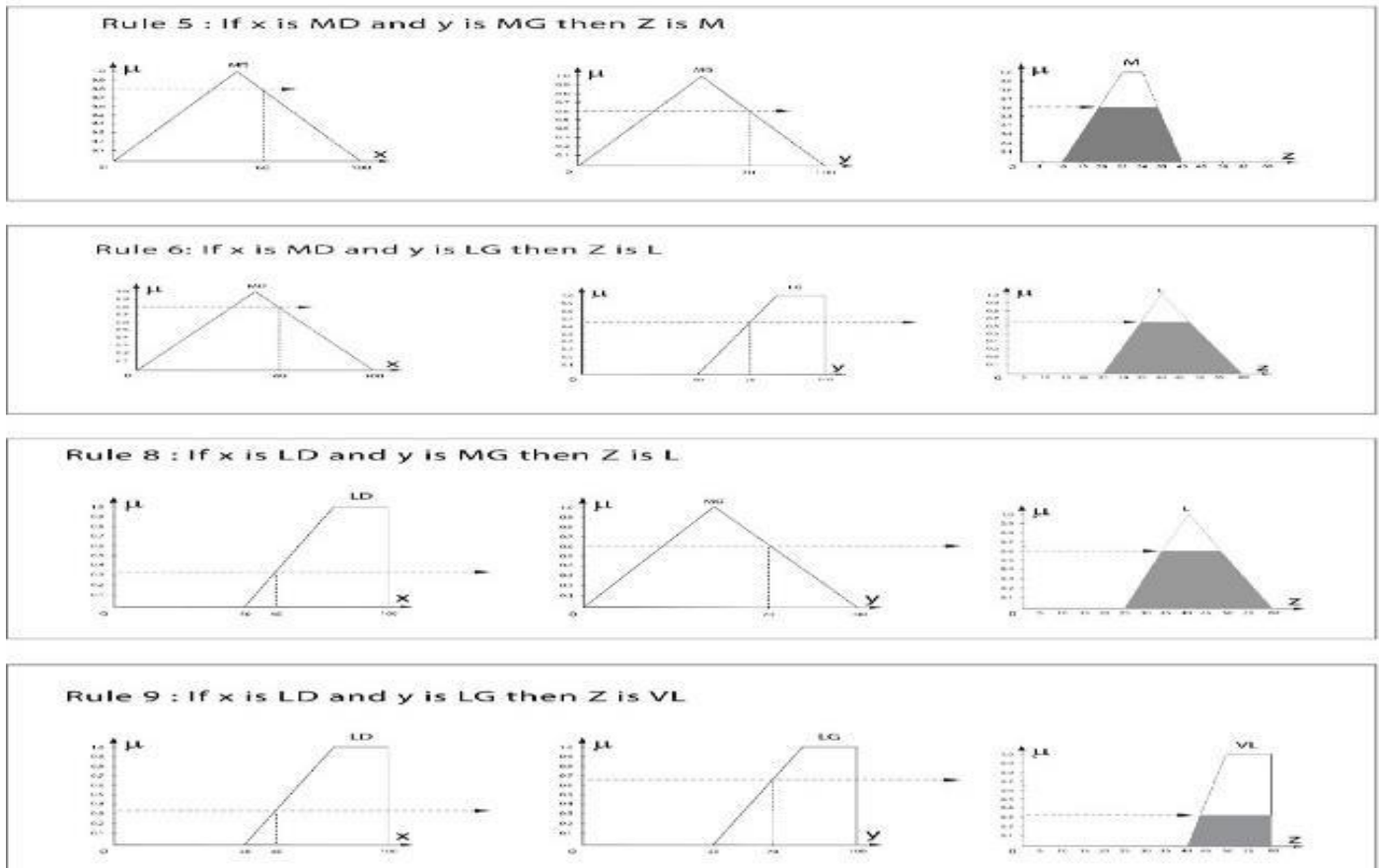

Fig. 16. The aggregated the control output for mixed FN (Combine TFN \& TrFN). 


\section{Defuzzification}

A crisp number is obtained from a fuzzy number during the execution of the aggregation process in a defuzzification process. The most widespread defuzzification technique is that the centroid technique. It finds a point representing the center of Gravity (COG), Center of Sum (COS) aggregated fuzzy set. A reasonable estimation can be obtained by calculating it over a simple of points. According to fig: 17, 18, 19, 20, 21, $22,23,24$ and 25 in our case results.

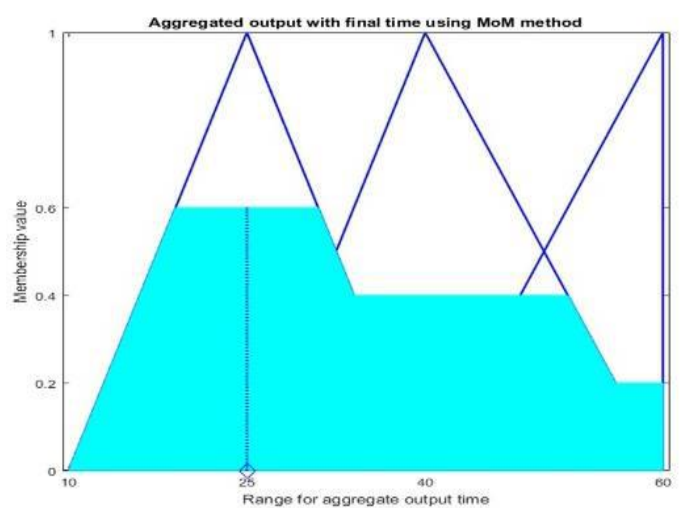

Fig. 17. Defuzzification output for FLC using the MOM method of TFN.

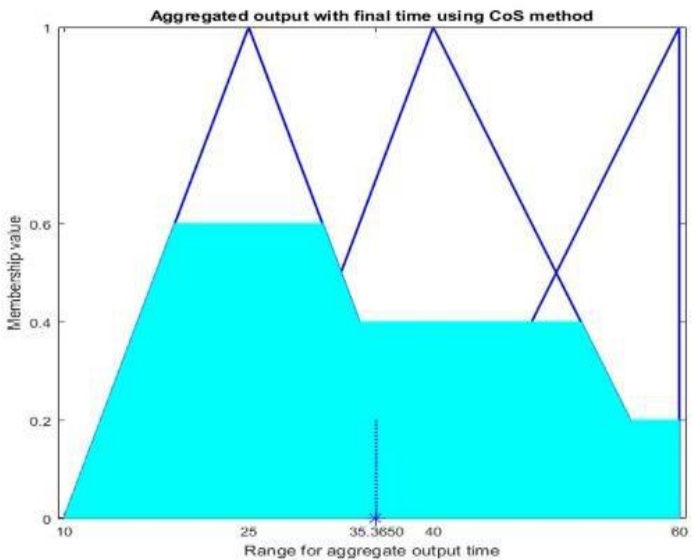

Fig. 18. Defuzzification output for FLC using the COS method of TFN.

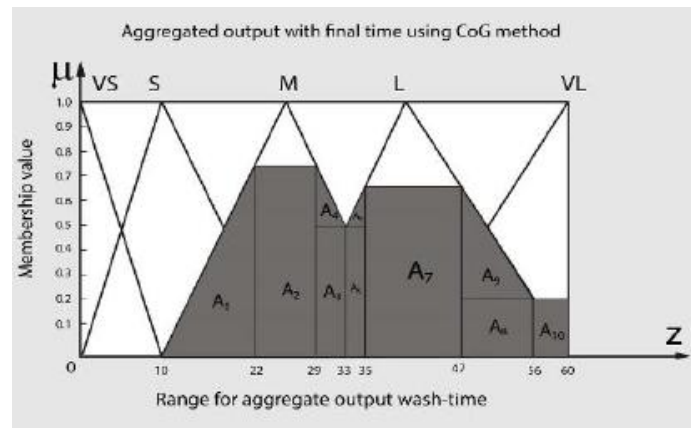

Fig. 19. Defuzzification output for FLC using the COG method of TFN

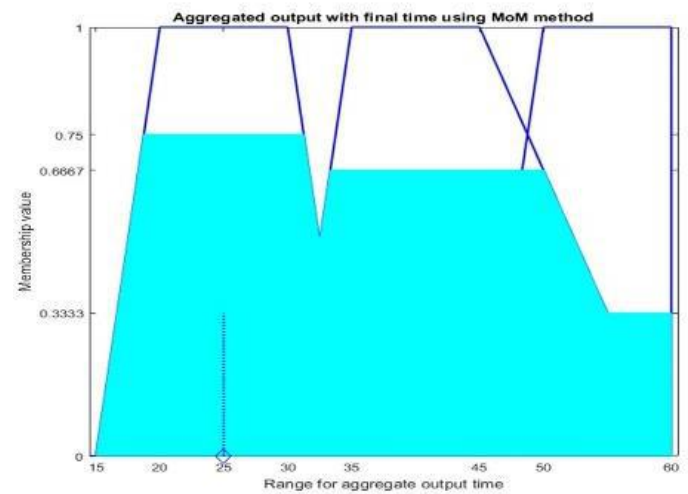

Fig. 20. Defuzzification output for FLC using the MOM method of TrFN.

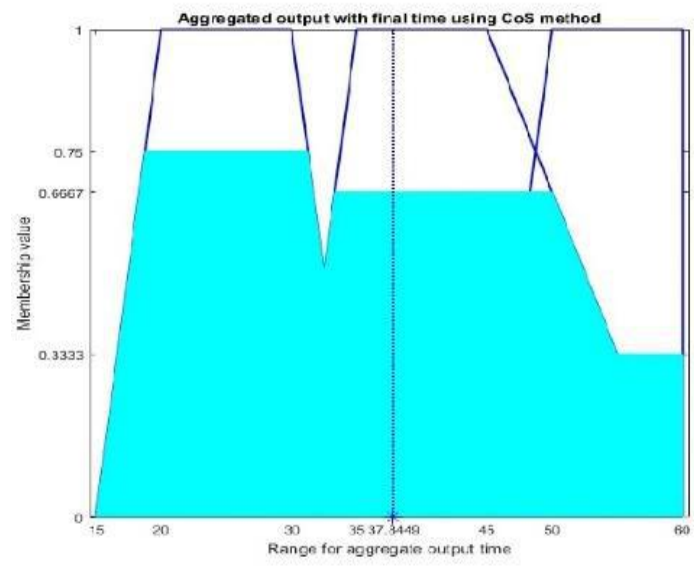

Fig. 21. Defuzzification output for FLC using the COS method of TrFN. 


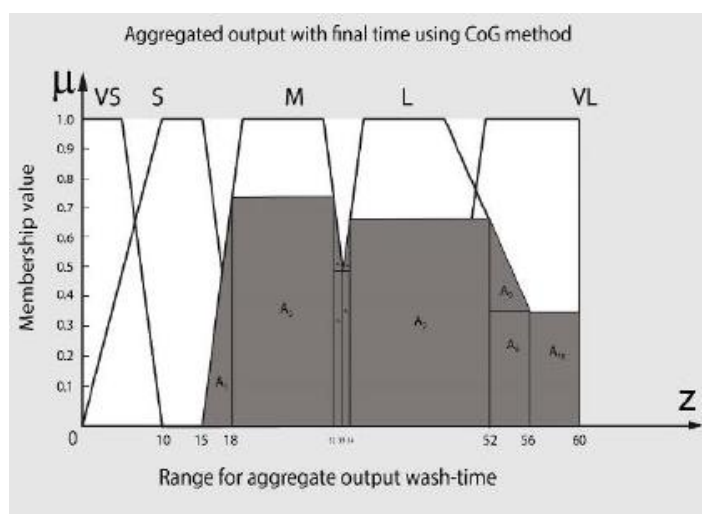

Fig. 22. Defuzzification output for FLC using the COG method of TrFN.

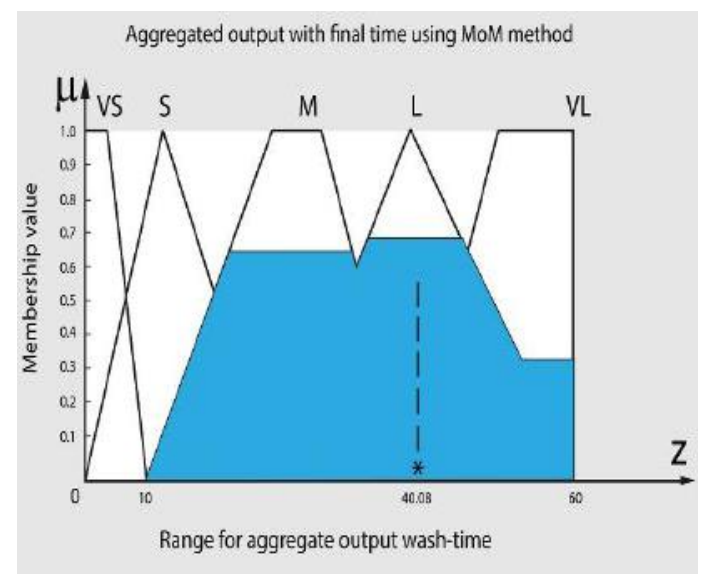

Fig. 23. Defuzzification output for FLC using the MOM method of mixed FN.

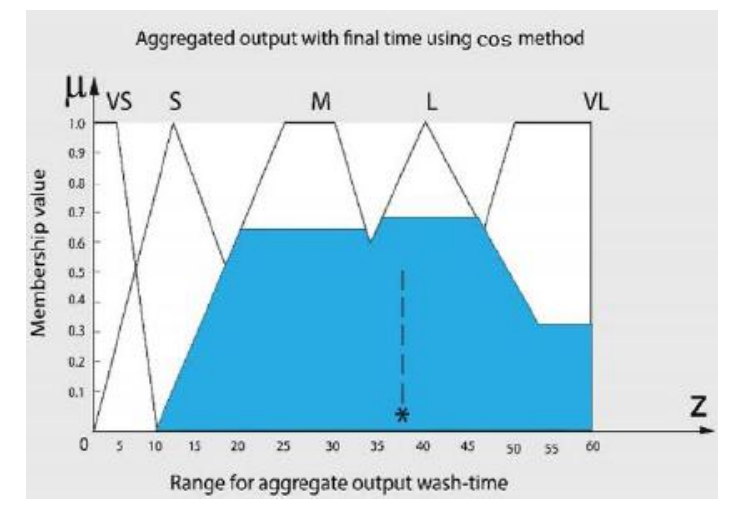

Fig. 24. Defuzzification output for FLC using the COS method of mixed FN.

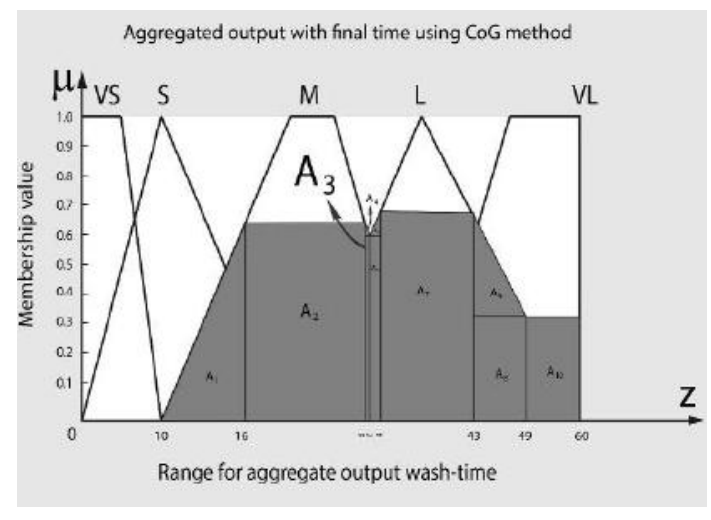

Fig. 25. Defuzzification output for FLC using the COG method of mixed FN.

\section{Result and Discussion}

Based on the present degree of dirt and the present amount of grease on the clothes, it can be said that this method will have different wash times. Here, a large number of values of dirt and grease can be used to evaluate the difference between these methods. Clearly, it can be said that these three techniques shown in the above tables which is used for comparison, provides wash time as output. Hence, by using "if" and "then" rules during the defuzzification of the washing machine, its output can be modified and verified at the same time. From table 4 . We can see that:

Using the Mean of Maximum (MOM) method, it can be seen that the wash time is the same for the triangular and trapezoidal fuzzy number but the difference of the wash time is very different for the mixed fuzzy number (i.e., Triangular and trapezoidal fuzzy 
Islam et al./J. Bangladesh Acad. Sci. 45(1); 105-116: June 2021

\begin{tabular}{cccccc}
\hline Type of FN & $\begin{array}{c}\text { Type-Dirt } \\
(\boldsymbol{\%})\end{array}$ & $\begin{array}{c}\text { Amount Grease } \\
(\boldsymbol{\%})\end{array}$ & \multicolumn{3}{c}{ Wash-Time } \\
\cline { 4 - 6 } & & & COG & COS & MOM \\
\hline TFN & 60 & 70 & 33.650 & 33.365 & 25 \\
TrFN & 60 & 70 & 34.30 & 35.3650 & 25 \\
Mixed FN & 60 & 70 & 34.30 & 35.3650 & 40.0835 \\
\hline
\end{tabular}

number). So, the mean of maximum method's use is reasonable in triangular and trapezoidal fuzzy numbers.

Again, using the Center of Sum (COS) method, it can be explained that wash time is located between triangular trapezoidal and mixed fuzzy numbers. So, in the case of mixed fuzzy numbers, it is advisable to use the COS method. Also, using the Center of Gravity (COG) method, it can be seen that wash time is found between triangular, trapezoidal, and mixed fuzzy numbers. Therefore, it is reasonable to use the COG method for mixed fuzzy numbers.

From the above discussion, we can say that the power consumption will be lowest in triangular and trapezoidal fuzzy numbers using the MOM method. On the other hand, when using the MOM method, wash time is the highest used for mixed fuzzy numbers. So, it is best to avoid this method.

\section{Conclusion}

Mathematicians use only the triangular or trapezoidal fuzzy number in the models used to find the wash time of the washing machine. In our mathematical research, we have calculated the wash time using the Center of Gravity (COG), the Center of Sum (COS), and the Mean of Maxima (MOM) models with the help of triangular, trapezoidal, and mixed fuzzy numbers and discussed their comparison.

From the above discussion, we have shown that the difference in wash time available in different models when using a mixed fuzzy number is less than the wash time available when using the other two fuzzy numbers in that model. Therefore, our research concludes that the use of mixed fuzzy numbers in calculating wash time is more reasonable than triangular and trapezoidal fuzzy numbers.

\section{Acknowledgment}

The authors of this paper are thankful to other authors whose names are included in the references section for their suggestions which helps us to modify this paper. Besides, we are also grateful to the Department of Mathematics, Faculty of Science, University of Rajshahi for their unconditional support and cooperation.

\section{References}

Aggarwal M. Fuzzy logic controller for washing machine. IIT Kharagpur. 2011.

Akram M, Habib S and Javed I. Intuitionistic fuzzy logic control for washing machines. Indian J. Sci. Technol. 2014; 7 (5); 654.

Alhanjouri MA and Ahmed A. Optimize wash time of washing machine using fuzzy 
logic, optimize wash time of washing machine using fuzzy logic. 2013.

Amin MR, Hossain MS and Miah SS. Fuzzy pairwise regular bitopological spaces in quasi-coincidence sense. J. Bangladesh Acad. Sci. 2020; 44(2); 139-143.

Bai Y and Wang D. Fundamentals of fuzzy logic control-fuzzy sets, fuzzy rules and defuzzifications, In: Advanced fuzzy logic technologies in industrial applications, Springer. 2006; pp. 17-36.

Dahmardeh $\mathrm{N}$ and Pourshahabi V. Agility evaluation in public sector using fuzzy logic. Iran J. Fuzzy Syst. 2011; 8(3); 95-111.

Isabels $\mathrm{K}$ and Uthra G. An application of linguistic variables in assignment problem with fuzzy costs. Int. J. Comput. Eng. Sci. 2012; 2(4): 1065-1069.

Mahbub MA, Hossain MS and Hossain MA. On Q-Compactness in Intuitionistic Fuzzy Topological Spaces. J. Bangladesh Acad. Sci. 2019; 43(2); 197-203.
Mamdani EH. Application of fuzzy algorithms for control of simple dynamic plant. Proc. Inst. Electr. Eng. IET. 1974; 121: 1585-1588.

Miah SSA, Amin R and Rana S. Fuzzy normal topological space in quasi-coincidence sense. J. Bangladesh Acad. Sci. 2018; 42(1); 201-205.

Mogharreban N and Dilalla L. Comparison of defuzzification techniques for analysis of non-interval data. In: NAFIPS 2006-2006 Annual Meeting of the North American Fuzzy Information Processing Society, IEEE. 2006; pp. 257-260.

Mukaidono M. Fuzzy logic for beginners. World Sci. 2001.

Saade JJ and Diab HB. Defuzzification methods and new techniques for fuzzy controllers. 2004.

Zadeh LA. Fuzzy sets. Inf. Control. 1965; 8 (3); 338- 353. 